\title{
A Point-to-Point Protocol Improvement to Reduce Data Call Setup Latency in Cdma2000 System
}

\author{
Eun-sook Lee ${ }^{1}, \mathrm{Kyu}-\mathrm{seob} \mathrm{Cho}^{1}$, and Sung Kim ${ }^{2}$ \\ ${ }^{1}$ School of Information \& Communication Engineering, SungKyunKwan University, \#300, \\ Chunchun-dong, Jangan-gu, Suwon, Republic of Korea \\ riya213@skku.edu, kscho103@yurim.skku.ac.kr \\ ${ }^{2}$ Network R\&D center, SK Telecom Co., \#11, Ulgiro1-ga, Joong-gu, Seoul, Republic of Korea \\ solar1@sktelecom.com
}

\begin{abstract}
The wireless cellular networks have evolved from IS-95A/B to cdma2000 1X, EV-DO, and WCDMA to improve data service quality. A carries also preserve in their efforts to rev up wireless data service. Despite this efforts, call setup latency has occurred on cdma2000 1X and EV-DO systems. It is an impediment to data service activation. In measuring the real delay time during call establishment from MS to BSC, PCF and PDSN, it is found out that point-to-point protocol between MS and PDSN is major source of delay. Therefore, a simplified PPP is proposed, considering the difference in transmission speed of links among nodes. Then the inter-working scenarios between MS and PDSN is presented with simplified PPP and/or legacy PPP, and the proposed scheme is verified with superior performance over legacy PPP, through comparing the number of packets required for data call setup.
\end{abstract}

Keywords: Simplified PPP, Call setup latency, Cdma2000 system, Wireless packet data service.

\section{Introduction}

The paradigm of mobile cellular service has shifted from voice to packet data and a wireless carries have researched and developed a radio technology to provide higher speed data rate, handoff to decrease packet loss rate and wireless TCP considering radio characteristics with burst error. They make efforts to improve an infrastructure to meet a service quality criteria and requirements clarified in ITU-Y.154. It is downstream and upstream transmission rate, packet loss rate, end-to-end packet transmission delay and packet loss rate during handoff on mobile cellular network.

The wireless carriers operating cdma2000 system come to realize that the data call setup time in the system is inferior to General Packet Radio Service (GPRS), and is a key factor in increasing data service usage as well as user friendly UI (user Interface), various contents, billing policy to reduce the tariff burden, and strong security. Users using high speed Internet have not experienced any call setup delay. Even for the data service on the cdma2000 network, these users also want similar level services for data throughput, call setup latency and contents, and so on. When these users attempt the 
wireless data service with their mobile phone, they may abandon the service trial if the call setup latency is much longer than the wired Internet.

Through the measurement and analysis of the delay time from Mobile Station (MS) to Base Station (BS), Packet Control Function (PCF) and Packet Data Serving Node (PDSN) on real network, it is concluded that Point-to-Point Protocol (PPP) operations between MS and PDSN were major source of the delay. A PPP is based on peer-topeer mechanism is to establish a data link between MS and PDSN. Generally PDSN takes more time to activate the PPP than MS. Because MS can start the PPP as soon as a traffic channel on radio assigns but PDSN has to wait to start it until A8 and A10 session are established. Thus PDSN could lose the first PPP packet from MS. Consequently, this peer-to-peer mechanism requires greater than 1.5 times the number of packets than when two end stations are defined as network and terminal side. In order to solve this problem it is necessary to decrease the number of packets considering the difference in transmission speed of links among nodes.

This paper is organized as follows. In Section II, cdma2000 network model is presented. Section III describes PPP operations for connection establishment. In addition, some of PPP's inherent problems related in call setup latency in cdam2000 system are examined. In Section IV, an improved PPP operation and the state transition diagram to solve setup latency, called simplified PPP (S-PPP), are defined. In section V and VI describe inter-working scenarios between MS and PDSN with S-PPP and/or legacy PPP in cdma2000 system and compare the performances.

\section{Cdma2000 Network Model}

Figure 1 shows the cdma2000 network model for Simple IP and Mobile IP service. Simple IP refers to a service in which an MS is assigned IP address and is provided IP routing service by an access provider network. Mobile IP refers to a service in which the user is able to maintain a persistent IP address even when handing off between RNs (Radio Networks) connected to different PDSNs [1].

The PPP, which is data link protocol, is located on Medium Access Control/ Link Access Control (MAC/LAC) layer in MS and Generic Routing Encapsulation (GRE) in PDSN. The PPP supports a multiplexing of network protocols and link configluration, error detection and value-added communication features such as compression

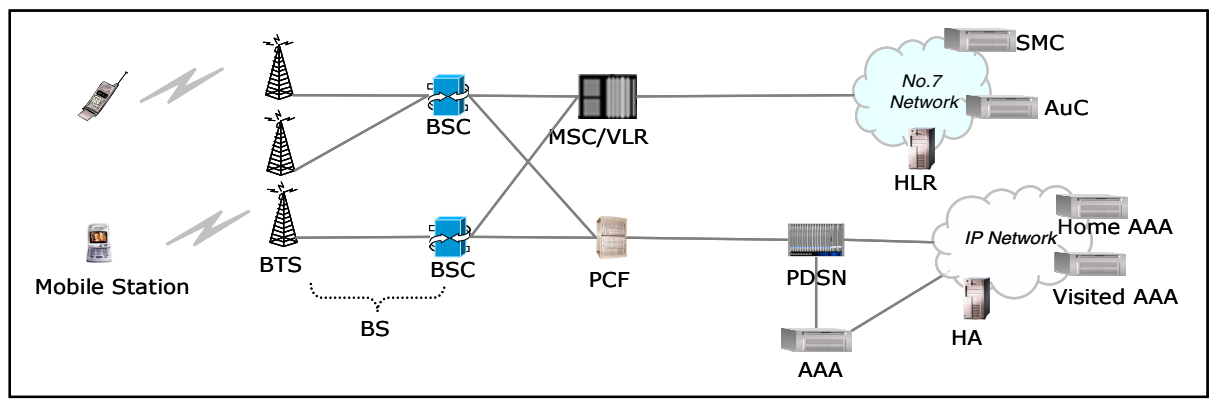

Fig. 1. Cdma2000 Network Reference Model 


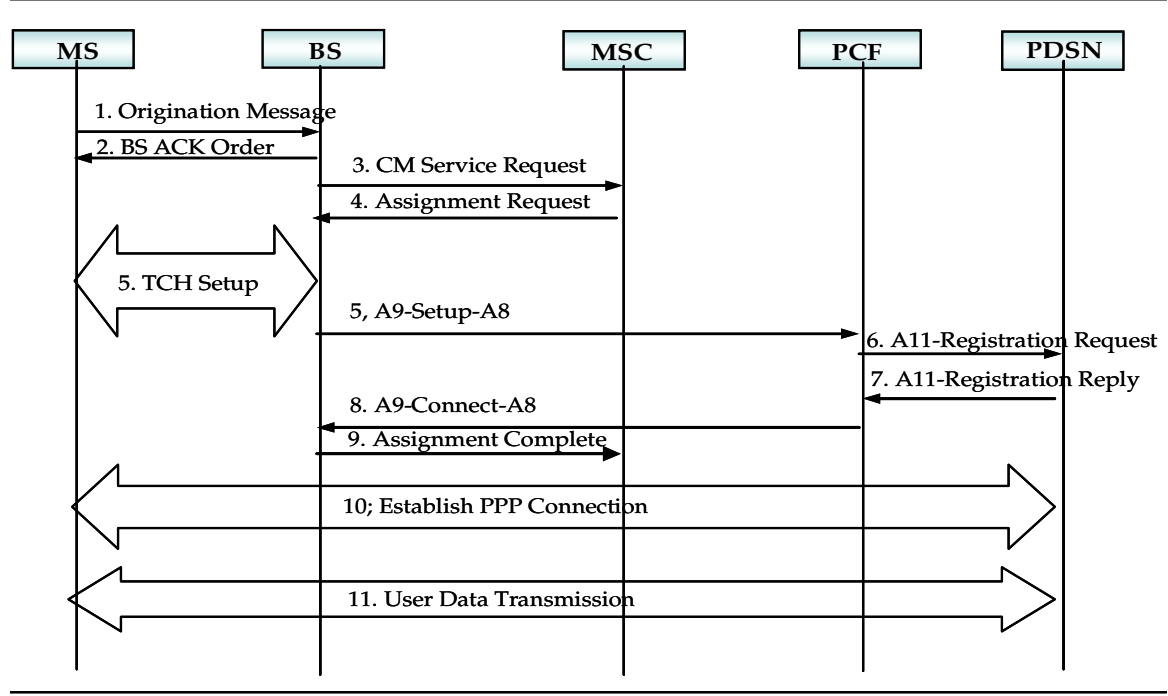

Fig. 2. Cdma2000 Packet Data Service Flows

and encryption, establishing network addresses and authentication [2]. The PDSN assigns IP addresses, transfers it through PPP to MS for Simple IP service and operates as Foreign Agent (FA) for Mobile IP service.

Figure 2 shows call establishment procedure for packet data service initiated by MS in idle and inactive state. The MS sends Origination message to the serving Base Station (BS), which include Service Option, Service instance discriminator and Indicator requesting acknowledgement of layer 2 on an access channel (step 1). The serving BS performs authentication by MSID through the serving MSC interconnec-tion. After positive authentication, the MS assigns radio resource (step 2 to 5). A8 session is established between the serving BS and the PCF using A9 signaling and A10 session between the serving PCF and the PDSN using A11 signaling (step 6 to 9). The procedures of PPP (step 11) are performed in order after completion of A10 session between the MS and the serving PDSN, which are Link Control Protocol (LCP), user authentication and Network Control Protocol (NCP). The MS can send/receive IP packets after PPP establishment (step 12) [3][4].

\section{PPP Operations on Cdma2000 Network}

The PPP is used widely regardless physical transmission link and transmission rate, and provides compatibility with almost all network technology. Therefore the PPP is utilized on wireless cellular system as link layer protocol between MS and PDSN and is recommended on 3GPP2 'wireless IP network' specification. It would deal with the LCP and IPCP operation included in the PPP in detail and survey the real usage in commercial network [1][2]. 


\subsection{LCP Operations}

LCP is to establish, terminate, and maintain data link connections as depicted in Figure 3.

Step 1 \& 2. - The serving PDSN sends a Configure-Request packet to the MS to negotiate link configuration after establishment of the A8 and A10 session. The MS also sends a Configure-Request packet to negotiate link configuration after the traffic channel assignment negotiates with BS. The step 2 may be executed prior to step 1. If the serving PDSN receives a Configure-Request before completion of the A10 session, the PDSN discards it.

Step $3 \& 4$. - The serving PDSN and the MS respond with a Configure-Ack. packet to each peer for reception of a Configure-Request with an acceptable set of configuration options. Upon completion of LCP establishment, the MS and/or the serving PDSN can perform authentication or NCP procedure.

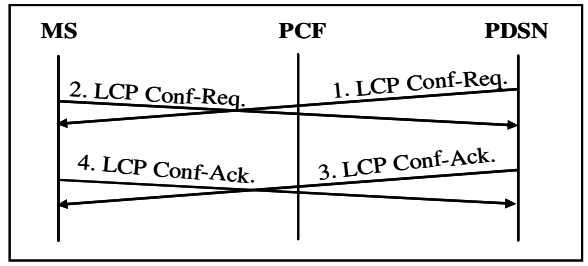

Fig. 3. LCP procedure

\subsection{IPCP Operations}

The IPCP in RFC 1332, which is one of the NCPs, is the most common and negotiates IP addresses and other parameters to configure, enable and disable the IP network protocol. The IPCP uses the same mechanism as the LCP to exchange packets.

Simple IP service for IPv4. Figure $4 \mathrm{a}$ is that the MS requests Simple IP service for IPv4 [1][5].

Step 1\& 2. - The serving PDSN sends a Configure-Request packet to the MS with IP Address option for itself after completion of the LCP establishment and positive authentication optionally. The MS also sends a Configure-Request packet to the serving PDSN with IP Address of 0.0.0.0, primary Domain Name System (DNS) address, and secondary DNS address regardless of IPCP state on the serving PDSN.

Step 3. - The serving PDSN assigns the MS an IP address for Simple IP service when the IP Address configuration option in Configure-Request received is a zero. The serving PDSN sends Configure-Nak. packet including new IP address of the MS. It is case that the serving PDSN accepts the DNS address from the MS. 
Step 4. - The MS acknowledges the Configure-Request with acceptable a specific compression protocol and IP Address configuration option.

Step 5. - The MS sends a Configure-Request to the serving PDSN after replacing IP Address option with new IP Address received from the PDSN.

Step 6. - The PDSN acknowledges the Configure-Request with acceptable set ofconfiguration options and transits to an 'Opened' state.

Simple IP service for IPv6. Figure $4 b$ is that the MS requests Simple IP service for IPv6 [1][6].

Step 1. -Because the serving PDSNs do not know the operation that MS requests. Thus, the serving PDSN sends an identical Configure-Request packet to initiate Simple IP service for IPv4 to the MS as in the former case.

Step 2. - The MS sends an IPv6CP Configure-Request packet to request Simple IP service for IPv6 to the PDSN with tentative IF-ID (Interface-Identifier) after completion of the LCP and positive authentication regardless of the PDSN state.

Step 3. - The serving PDSN recognizes the service when receives the ConfigureRequest's configuration options from the MS and responds with an IPv6CP Configure-Nak. packet including IF-ID to be able to construct the link-local IPv6 address and global IPv6 address at the MS.

Step 4. - The MS sends a Protocol-Reject packet to the serving PDSN and discontinues IPv4CP processing, because the MS of IPv6 mode can not analyze the Configure-Request for IPv4 from PDSN (step 1).

Step 5. - The serving PDSN requests IPv6CP establishment by sending an IPv6CP Configure-Request packet with IF-ID and specific compression protocol.

Step 6. - The MS sends the IPv6CP Configure-Request packet to the serving PDSN after replacing tentative IF-ID option with a new one received from the PDSN.

Step 7. - The PDSN acknowledges for the IPv6CP Configure-Request with acceptable set of configuration options and transits to an 'Opened' state.

Step 8. - The MS also responds with an IPv6CP Configure-Ack. packet for IPv6CP Configure-Request with acceptable set of configuration options to the PDSN and transits to an 'Opened' state.

Mobile IP service for IPv4. Fig. 4c is that the MS initiates Mobile IP service for IPv4 [1][5].

Step 1. - The serving PDSN sends a Configure-Request packet to the MS as the former case.

Step 2. - In order to request Mobile IP service, the MS sends a Configure-Request packet to access network without an IP Address option after completion of the LCP and positive authentication regardless of IPCP state on the serving PDSN.

Step 3. - The serving PDSN acknowledges for the Configure-Request packet with acceptable set of configuration options and transits to an 'Opened' state.

Step 4. - The MS also responds with a Configure-Ack. packet as positive response to the serving PDSN and transits to an 'Opened' state. 
After step 4, Mobile IP procedures for mobility management are performed. Both sides of a connection initiate the session establishment shown as dual procedures, because PPP is a peer-to-peer protocol. It might make the whole process unnecessarily complicated. In LCP operations, the MS can send a Configure-Request packet as soon as a traffic channel on radio assigns. The LCP packet could be sent to the serving PDSN before the A8 and A10 session establish, thus it could be lost between BS and PDSN. In this case, the restart timer on the MS expires and then the MS shall retransmit the Configure-Request packet after 3 seconds. Because the default restart timer is 3 seconds. Whenever the timer expires on the MS, the call setup latency becomes longer as much as the timer. The case can be seen several times on the traces of the call setup procedures in the real network. This is because the MS takes a shorter time than PDSN to reach the LCP ready state because of difference in transmission speed of links among nodes.

The purpose of the LCP operation is that the MS wants to set up a bidirectional data link layer session between it and PDSN for packet data services. Considering the roles of the MS and PDSN for its requirements and cddma2000 system characteristics, a simplified asymmetrical process can be designed for the LCP.

The similar arguments might be applied to the IPCP operation. It is excessively complicated because of the peer-to-peer properties and the various kinds of service that should be served. Unnecessary packets are included. For example, the first Configure-Request packet sent by PDSN is useless for IPv6 operation.

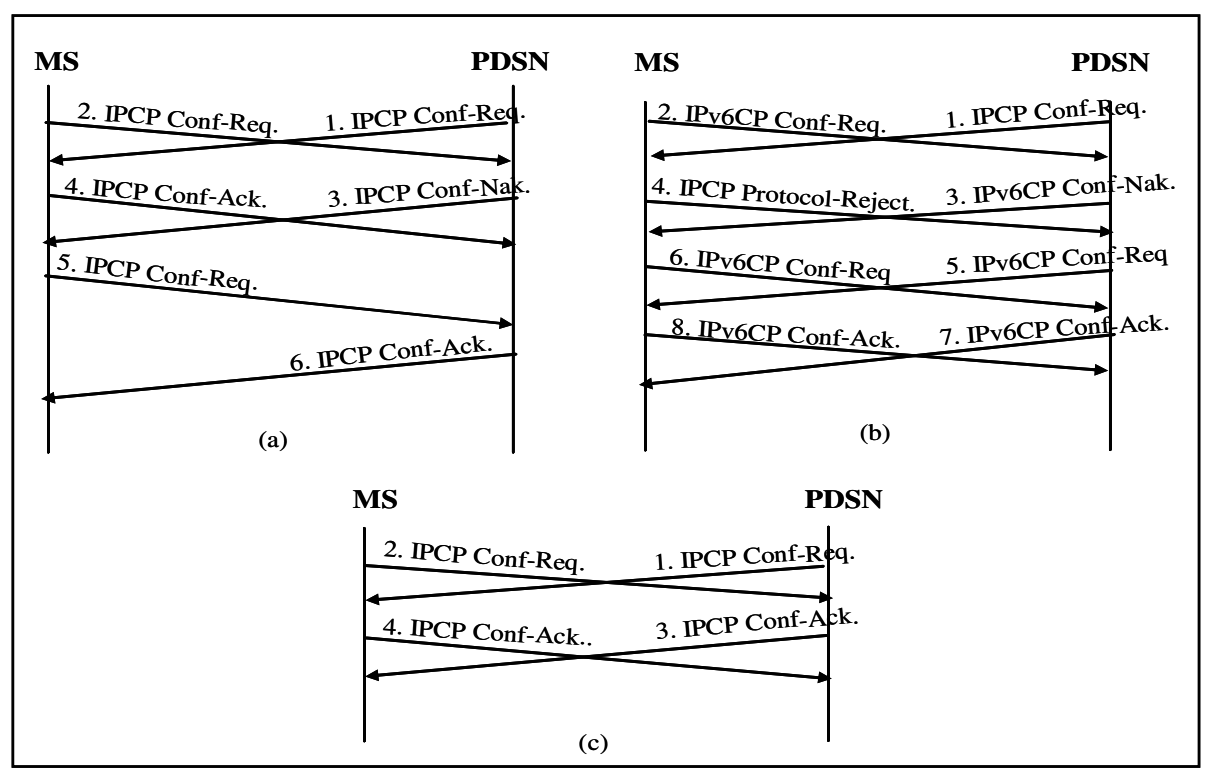

Fig. 4. IPCP procedure for a) Simple IP operation for IPv4; b) Simple IP operation for IPv6; c) Mobile IP operation for IPv4 


\section{Simplified PPP Proposal}

Through the field test measurement and log analysis, simplified PPP (S-PPP) is pro posed for the cdma2000 packet data service. For the packet data service, the MS always initiates the call setup process. PDSN recognizes the call setup request from the MS by receiving A11 signaling. Normally it is PDSN's turn to respond to the request from the MS. If the process is designed based on such request/response call process by network and terminal side definition, not on the peer-to-peer relations, many dual procedures in Figure 3 and 4 can be omitted.

The frame of S-PPP follows High-level Data Link Control (HDLC) format recommended in RFC 1662 and PPP format in RFC 1661[2]. And the operation mechanism for LCP and IPCP state are discriminated network from terminal side to restrict a peer-to-peer characteristic partly like server and client.

\subsection{S-PPP Operations}

Figure 5 depicts a basic procedure of S-PPP comparing with legacy PPP to establi-sh and terminate a data link between MS and the serving PDSN in normal case.

LCP operations. The step 1 and 2 in Figure 5 conform to LCP operations.

Step 1. - The serving PDSN sends a Configure-Request packet to the MS as previous legacy LCP operation and starts a restart timer. But the MS shall wait until reception of the Configure-Request packet in S-PPP operation. A configuration options in this packet are same with legacy LCP.

Step 2. - The MS responds with a Configure-Ack. packet when it acknowledges the reception of the Configure-Request packet with an acceptable set of configuration options. If the configuration options are not acceptable, the MS selects a configuration options want to negotiate with the PDSN and inserts them in Configure-Reject/Nak. packet and send the packet to the PDSN. If the PDSN can accept the whole configuration options in the Configure-Request packet from the MS, it sends Configure-Request packet with the configuration options and start restart timer. Completion of LCP procedures, authentication is determined during LCP negotiation.

IPCP operations. IPCP operations for Simple IP is from step 4 to 7 and Mobile IP service is step 4 and step 7 in Figure 5.

Step 4. - The MS determines a proper type of packet and a configuration options. In case of Simple IP service for IPv4, the MS sends a Configure-Request packet set the PDSN and MS address in IP Addresses configuration option 0.0.0.0. For Simple IP service for IPv6, the MS sends an IPv6CP Configure-Request packet including a tentative IF-ID. For Mobile IP service for IPv4, the MS sends Configure-Request packet without IP Address or IP Addresses configuration option. Another configuration options are same with legacy IPCP.

Step 5. - The serving PDSN responds with a Configure-Nak. or an IPv6CP Configure-Nak. packet as a negative response, in order to notify the receiving 
Configure-Request packet with an unacceptable set of configuration options. Thus the serving PDSN to support Simple IP service includes itself and the MS address in IP Addresses configuration option for IPv4 and an IF-ID configuration option for IPv6.

Step 6. - The MS sends a Configure-Request or an IPv6CP Configure-Request packet again after replacing IP Address or IF-ID with it received from the serving PDSN.

Step 7. - The serving PDSN responds with a Configure-Ack. or IPv6CP ConfigureAck. packet after reception of the Configure-Request or the IPv6CP Configure-Request packet with an acceptable set of configuration options and transits to an 'Opened' state.

Step $8 \&$ 9. - After Completion of successful IPCP negotiation, the PDSN sends an Account-Request packet to Authentication, Authorization and Accounting (AAA) server to notify of a billing start and gets Account-Response from the AAA. And the MS and the PDSN can deliver user data.

Step 10. - When the user wants to stop the service, the MS sends a TerminateRequest packet to the serving PDSN.

Step 11. - The serving PDSN responds with a Terminate-Ack. packet and terminates the S-PPP session. The A10 and A8 session are then release through A11 and A9 signaling.

Step 12 - The serving PDSN sends an Accounting-Request to the AAA server to notify of a billing stop.

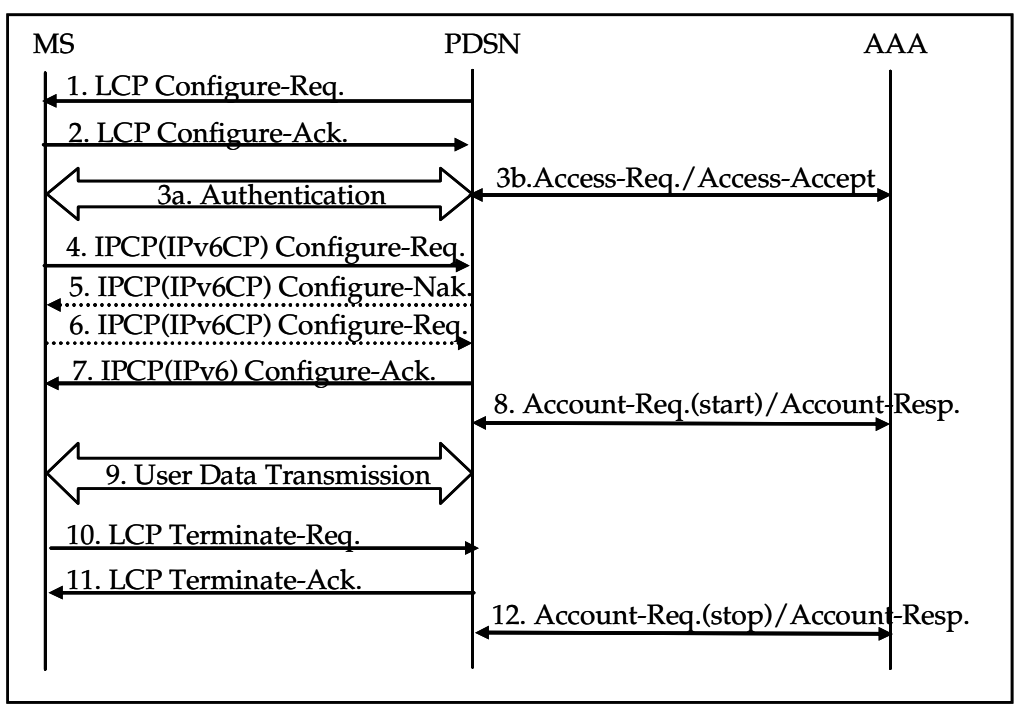

Fig. 5. Simplified PPP procedure

\subsection{State Transition Diagram of S-PPP}

In order to improve the PPP performance, a state transition diagram of S-PPP divides into network and terminal side. Figure 6a depicts the S-PPP state transition diagram 
on the PDSN for LCP operation and on the MS for IPCP operation. For instance the normal procedure for LCP operation is as follows: The 'Initial' state transits to the 'Req-Sent' via the 'Starting' after an administrative open is initiated and low layer is available and the serving PDSN sends a Configure-Request packet to peer.

After the PDSN receives a Configure-Ack. packet with an acceptable set of configuration options, it transits to an 'Opened' state. The 'Opened' state means that authentication or IPCP operation can process following the completion of LCP operation and user traffic can be delivered. In IPCP operation, above description is for the MS.

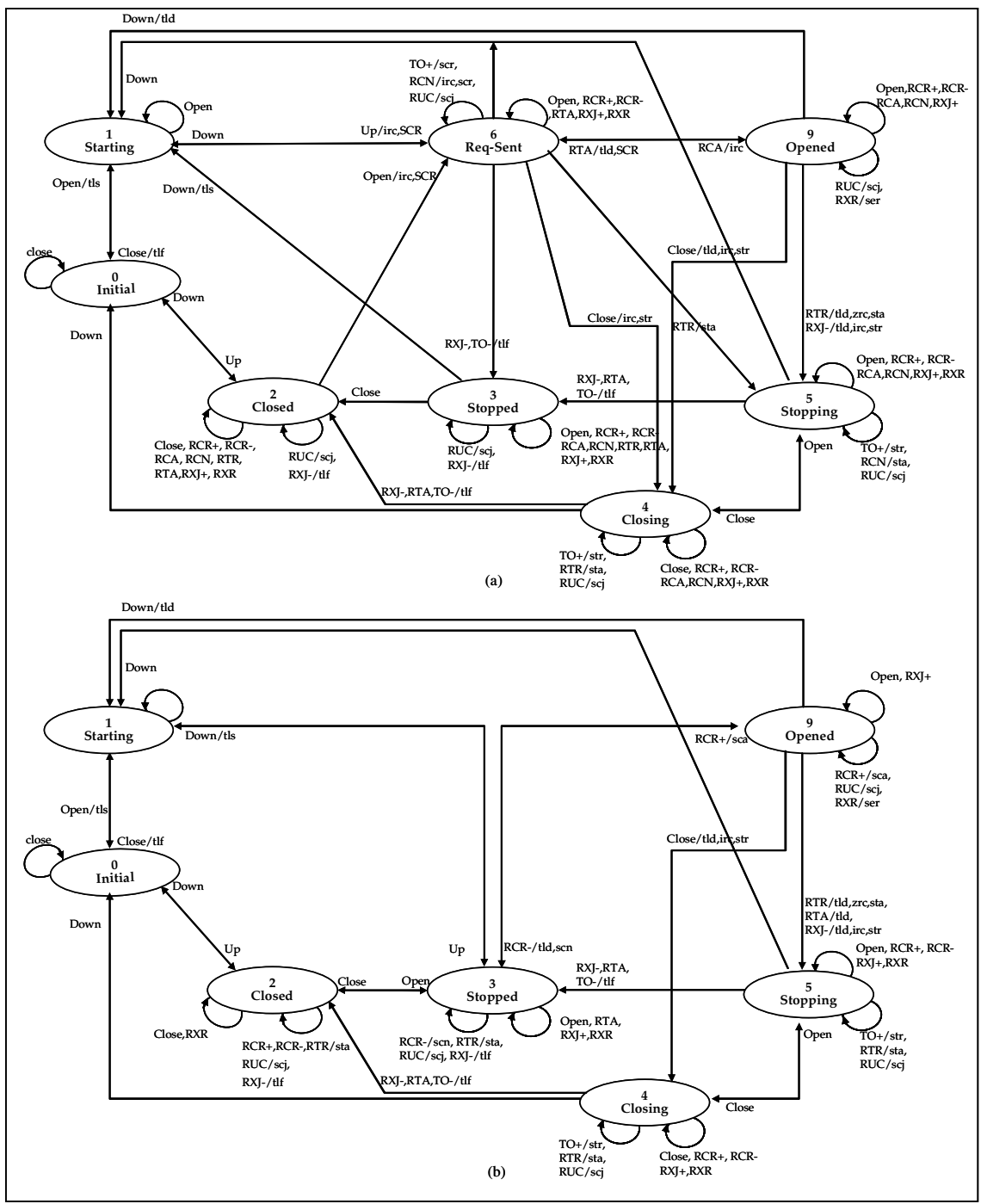

Fig. 6. State transition table for a) LCP operation on PDSN / IPCP operation on MS b) LCP operation on MS / IPCP operation on PDSN 
Figure $6 \mathrm{~b}$ is the S-PPP state transition diagram on the MS for LCP operation and on the PDSN for IPCP operation. For instance the normal procedure for LCP operation is as follows: The 'Initial' state transits to the 'Stopped' state via 'Starting' state after an administrative open is initiated and low layer is available. And the MS waits a Configure-Request packet from peer. After reception of this packet with an acceptable set of configuration options, the MS responds with a Configure-Ack. packet and transits to 'Opened' state. If not, the MS responds with a Configure-Reject or a Configure-Nak. packet and keeps the 'Stopped' state.

\section{S-PPP Inter-working Scenarios on Cdma2000 Network}

In order to implement the S-PPP on the existing cdma2000 network, the MS and the serving PDSN are required to know how to discriminate a type of PPP, such as legacy PPP and S-PPP, and how to notify a network capability regarding a type of PPP.

It is recommended that the Service Option parameter is able to get through the Origination message as a PPP discriminator. Using the Service Option is a better way to minimize the modification on network nodes and the MS than a new parameter. In the cdma2000 system, the Service Option 33 means packet data service for cdma2000 $1 \mathrm{X}$ system and 59 for EV-DO. The meaning of Service Option 33 will be expanded for cdma2000 1X packet data service using legacy PPP and 59 for EV-DO using legacy PPP. Thus a new Service Option 80 is defined, which means cdma2000 1X packet data service using S-PPP and the other new Service Option 81 for EV-DO using S-PPP as temporary value. According to the capability of the network and the MS to handle the PPP and/or S-PPP, there are six scenarios to inter-work as follows;

Scenario 1 : The MS and PDSN handle both legacy PPP and S-PPP.

Scenario 2 : The PDSN handles both legacy PPP and S-PPP and the MS handles only legacy PPP.

Scenario 3: The PDSN handles both legacy PPP and S-PPP and the MS handles only S-PPP.

Scenario 4: The PDSN handles only legacy PPP and MS handles both legacy PPP and S-PPP.

Scenario 5: The PDSN and the MS handles only legacy PPP.

Scenario 6: The PDSN handles only legacy PPP and MS handles only S-PPP.

The Scenario 4 and 5 follow the current packet data procedure because the PDSN operates as if it uses legacy PPP. Although the PDSN can inform the MS that it can handle S-PPP in Scenario 2, the MS cannot recognize the situation and it operates as if it was using Service Option 33 or 59, according to network model. In Scenario 6, it is impossible to provide packet data service. Only in the case of Scenario 1 and 3, SPPP can be activated using Service Option 80 or 81 , In order to notify the types of PPP that PDSN can handle, using Over The Air Service Provisioning (OTASP) is proposed [7]. The information carriers want to add to a customer handsets is delivered through SMDPP and OTASP Data Messages in Figure 7. In case of Scenario 1 and 3, the MS gets PDSN capability about the type of PPP through OTASP procedure 


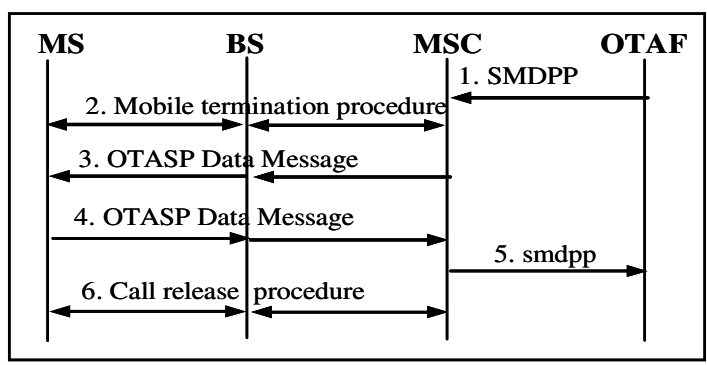

Fig. 7. PPP type notification through OTASP

(Figure 7). When the MS originates a data call on cdma2000, the procedure follows Figure 2 and 5 for S-PPP.

\section{Performance Evaluation}

The performance is compares by counting the number of packets exchanged between the MS and the PDSN. Table 1 shows the number of packets for a normal case and two abnormal cases in case the authentication is performed and omitted.

First, the normal case is that the legacy PPP operates according to the procedure in Figure 3 and 4 and S-PPP according to the procedure in Figure 5. Second, in abnormal case 1, it is assumed that the MS or the PDSN rejects the Configure-Request for LCP received from peer. Last, abnormal case 2 means that the MS or the PDSN rejects the two Configure-Request packets for LCP and IPCP.

Performing the authentication, $60 \%$ to $69.2 \%$ of the number of packets for legacy PPP operation are required for the S-PPP operation in the normal case. The packet

Table 1. Comparison of the number of packets between S-PPP and legacy PPP

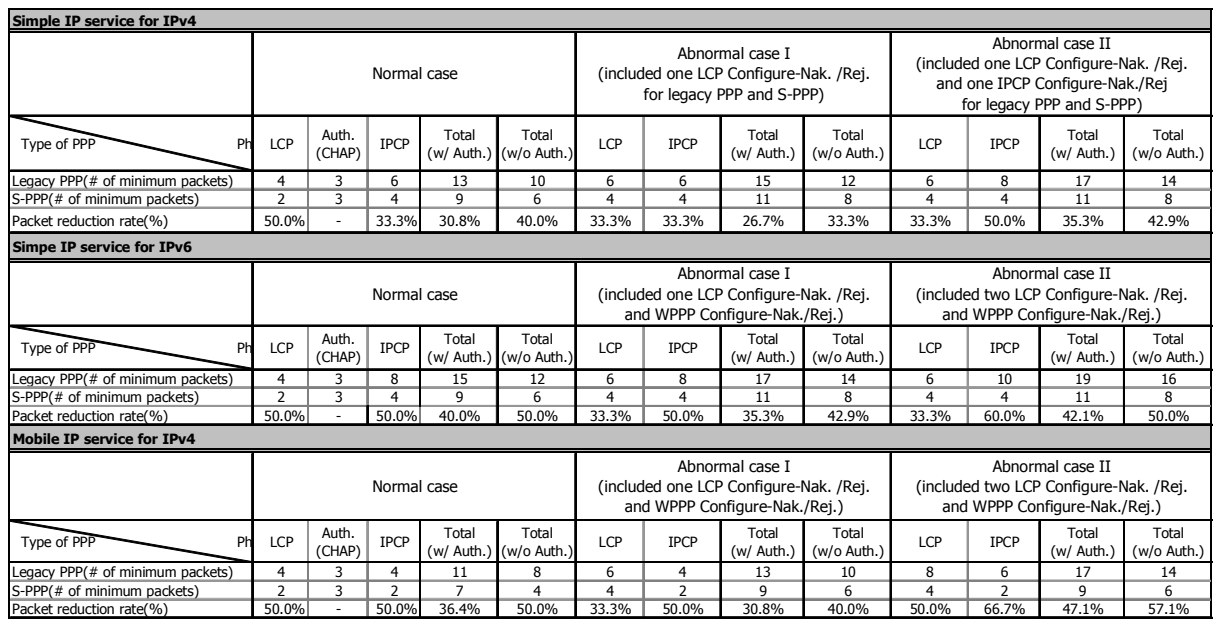


reduction rates are $26.7 \%$ to $35.3 \%$ in abnormal case 1 and $35.3 \%$ to $47.1 \%$ in abnormal case 2 .

And omitting the authentication, $50 \%$ to $60 \%$ of the number of packets for legacy PPP are required for the S-PPP in the normal case. The packet reduction rates are $33.3 \%$ to $42.9 \%$ in abnormal case 1 and $42.9 \%$ to $57.1 \%$ in abnormal case 2 .

\section{Conclusions}

The Call setup latency due to PPP becomes an obstacle for packet data service activation on the cdma2000 network. In this paper, an improved PPP is proposed, simplified PPP, as the result of the analysis on the RFC 1661, log file of call setup proce-dure in the real commercial network, and the specification for the cdma2000. In order to simplify the PPP procedure, considering their roles in the network, a simplified asymmetrical process adapts for the LCP and the IPCP operation. The number of packets is also reduced. To demonstrate how the proposed scheme could be applied to the current cdma2000 network, the inter-working scenarios between the MS and PDSN to be able to minimize the system changes are presented. The PDSN notifies the MS of the network capability about the type of PPP using OTASP, and in order to discriminate S-PPP and legacy PPP, a new Service Option value is defined. To evaluate the performance of the S-PPP, the number of packets and between the MS and the PDSN were compared. The S-PPP presents a packet reduction effected greater than $50 \%$.

\section{References}

1. 3GPP2, P.S0001-B v2.0. : CDMA2000 Wireless IP Network Standard. Sep., 2004

2. W. Simpson. : The Point-to-Point Protocol (PPP). RFC1661. July 1994.

3. 3GPP2 A.S0016-C v1.0. : Interoperability Specification (IOS) for cdma2000 Access Network Interfaces, Part 6(A8 and A9 Interfaces). Feb., 2005.

4. 3GPP2 A.S0017-C v1.0. : Interoperability Specification (IOS) for cdma2000 Access Network Interfaces, Part 7 (A10 and A11 Interfaces). Feb,. 2005.

5. G. McGrgor. : The PPP Internet Protocol Control Protocol (IPCP). RFC 1332. May 1992

6. D. Haskin and E.Allen. : IP Version 6 over PPP. RFC 2472. Dec., 1998

7. 3GPP2 C.S0016-C. : OTASP of MSs in Spread Spectrum Standards, Release C. Oct., 2004 